\title{
HEEGAARD SPLITTINGS AND SPLITTING HOMOMORPHISMS
}

\author{
BY \\ WILLIAM JACO( $\left.{ }^{1}\right)$
}

1. Introduction. Let $M$ be a closed, orientable three-manifold and suppose $(U, V)$ is a Heegaard splitting of $M$. Denote the common boundary of $U$ and $V$ by $S$. Let $u:\left(S, s_{0}\right) \rightarrow\left(U, s_{0}\right)$ and $v:\left(S, s_{0}\right) \rightarrow\left(V, s_{0}\right)$ be the inclusion maps. In [9] J. Stallings introduced a natural homomorphism to investigate; namely, the homomorphism

$$
\left(u_{*}, v_{*}\right): \pi_{1}\left(S, s_{0}\right) \rightarrow \pi_{1}\left(U, s_{0}\right) \times \pi_{1}\left(V, s_{0}\right)
$$

where $\left(u_{*}, v_{*}\right)(l)=\left(u_{*}(l), v_{*}(l)\right)$. The homomorphism $\left(u_{*}, v_{*}\right)$ is called the splitting homomorphism of $\pi_{1}\left(S, s_{0}\right)$ induced by $(U, V)$.

In studying the results and questions presented by Stallings in [9], a rather obvious question comes to mind. With the three-manifold $M$ and the Heegaard splitting $(U, V)$ of $M$ is related the algebraic configuration called the splitting homomorphism induced by $(U, V)$, is it possible that all such algebraic configurations occur in precisely this way? That is, suppose $S$ is a closed, orientable surface of genus $n>0$. Let $F_{1}$ and $F_{2}$ denote free groups of rank $n$ and suppose $\eta_{1}, \eta_{2}$ are homomorphisms of $\pi\left(S, s_{0}\right)$ onto $F_{1}$ and $F_{2}$, respectively. If

$$
\left(\eta_{1}, \eta_{2}\right): \pi_{1}\left(S, s_{0}\right) \rightarrow F_{1} \times F_{2}
$$

is the homomorphism defined as $\left(\eta_{1}, \eta_{2}\right)(l)=\left(\eta_{1}(l), \eta_{2}(l)\right)$, then is there a closed, orientable three-manifold $M$ and a Heegaard splitting $(U, V)$ of $M$ so that the splitting homomorphism induced by $(U, V)$ is (in some sense) $\left(\eta_{1}, \eta_{2}\right)$ ? In Theorem 5.2 this question is answered in the affirmative.

The homomorphism $\left(\eta_{1}, \eta_{2}\right)$, as described above, is called a splitting homomorphism. In Theorem 5.3, a one-one correspondence is established between equivalence classes of Heegaard splittings and equivalence classes of splitting homomorphisms.

In $\S 6$, it is shown that two conjectures made by J. Stallings in [9] are equivalent to Poincaré's Conjecture that a simply-connected, closed three-manifold is topologically equivalent to the three-sphere. These conjectures appear in $\$ 6$ as Conjecture B and Conjecture D (using the notation of [9]). It is interesting that Conjecture $\mathrm{D}$ can be stated using only the language of algebra.

Received by the editors October 14, 1968.

(1) This paper is part of the author's doctoral dissertation written at the University of Wisconsin under the supervision of Professor D. R. McMillan, Jr. The author was supported by the Wisconsin Graduate Research Committee-WARF Funds. 
The proofs of Theorem 5.2 and Theorem 5.3 are dependent on the study of mappings of closed surfaces into wedges given in $\$ 3$ and $\$ 4$. Using techniques of surgery on PL maps, it is shown that certain mappings of closed surfaces into wedges have mapping cylinders which are handlebodies.

Perhaps of independent interest is Corollary 3.3 of $\$ 3$. It states that there is a homomorphism of the fundamental group of a closed surface with Euler characteristic $2-p$ onto a free group of rank $k$ if and only if $k \leqq[p / 2]$ where [p/2] is the greatest integer less than or equal to $p / 2$.

The basic definitions appear in $\$ 2$. Some other definitions appear in the particular sections in which they are used.

2. Basic definitions and notation. The term map is used to mean continuous function. Let $I$ denote the closed unit interval $[0,1]$. Suppose $f: S \rightarrow X$ is a map. Consider the disjoint union of $S \times I$ and $X$ and introduce the equivalence relation $s \times 1 \sim f(s)$ for each $s \in S$. The identification space [2] of this relation is called the mapping cylinder of $f$ and is denoted $C(f)$.

The notation of simplex, complex, simplicial map, etc. may be found in [2, Chapter 1]. If $K$ is a complex, then the Euler characteristic of $K$ is denoted $\chi(K)$. A map of the underlying point set of a complex $K$ into the underlying point set of a complex $L$ is called a piecewise linear (PL) map if and only if there is a subdivision $K^{\prime}$ of $K$ and a subdivision $L^{\prime}$ of $L$ so that the map is simplicial from $K^{\prime}$ to $L^{\prime}$.

If $X$ is a space and $x_{0}$ is a point of $X$, then for $k \geqq 1, \pi_{k}\left(X, x_{0}\right)$ denotes the $k$ th homotopy group of $X$ based at $x_{0}$ [1]. The group $\pi_{1}\left(X, x_{0}\right)$ is also called the fundamental group of $X$ based at $x_{0}$. If $X$ is arcwise connected, then the $k$ th homotopy group is sometimes simply written $\pi_{k}(X)$. Suppose $f$ is a map from $\left(S, s_{0}\right)$ to $\left(X, x_{0}\right)$, then the homomorphism of $\pi_{1}\left(S, s_{0}\right)$ into $\pi_{1}\left(X, x_{0}\right)$ induced by $f$ is denoted $f_{*}$.

An $n$-manifold is a connected metric space in which each point has a neighborhood homeomorphic to the closed unit ball in $E^{n}$. If $M$ is an $n$-manifold, then the interior of $M$ (Int $M$ ) is the set of points of $M$ which have an open neighborhood homeomorphic to the interior of the unit ball in $E^{n}$. The boundary of $M(\operatorname{Bd} M)$ is defined to be $M$-Int $M$. If $M$ is a compact $n$-manifold and $\mathrm{Bd} M=\varnothing$, then $M$ is said to be a closed n-manifold. The term surface is synonomous with 2-manifold. A three-manifold is called a handlebody of genus $n, n \geqq 0$, if it is a regular neighborhood of a finite, connected graph with Euler characteristic $1-n$. In the case that the handlebody is orientable, it is often referred to as a cube-with-handles of genus $n$. The term regular neighborhood is used in the sense of [14, Chapter 3].

Sometimes it is convenient to denote the point set interior of a subset $Y$ of a space $X$ by $Y^{0}$, where there can be no confusion that this refers to the topology of $X$. An $n$-manifold $M$ is said to be simply connected if $\pi_{1}(M)$ is the trivial group.

For definitions of free group, direct product of groups, and free product of groups, the reader is referred to [4].

3. Mapping surfaces into wedges. Let $T$ denote a wedge at $t_{0}$ of $k$ simple closed 
curves $T_{1}, \ldots, T_{k}$. In this section a study of mappings of closed surfaces into $T$ is considered. Let $x$ denote a point of $T$ distinct from $t_{0}$. Then there is an embedding $\phi$ of $x \times[-1,1]$ into $T$ so that $\phi(x, 0)=x$. Define $U(x)=\phi(x \times[-1,1])$. Then $U^{+}(x)=\phi(x \times[0,1])$ is called the positive side of $x$ in $U(x)$ while

$$
U^{-}(x)=\phi(x \times[-1,0])
$$

is called the negative side of $x$ in $U(x)$.

A PL map $f$ of a closed surface $S$ into $T$ is said to be transverse with respect to $x$ if it is true that

(1) Each component of $f^{-1}(x)$ is a polyhedral simple closed curve in $S$ and

(2) there is a neighborhood $U(x)$ of $x$ in $T$, as above, so that for each component $J$ of $f^{-1}(x)$, the component $U(J)$ of $f^{-1}(U(x))$ containing $J$ is a product neighborhood of $J$ in $S$ where the parametrization of $U(J)$ as a product is that induced by $\phi$; i.e. the parametrization of $U(J)$ as a product may be realized by an embedding $\psi$ of $J \times[-1,1]$ into $S$ with $\psi(s, 0)=s$ for every $s \in J$ and with $f$ mapping each arc $\psi(s \times[-1,1])$ homeomorphically onto $\phi(f(s) \times[-1,1])$ where $f \circ \psi(s, r)=\phi(f(s), r)$, $-1 \leqq r \leqq 1$.

Suppose $\left\{x_{1}, \ldots, x_{p}\right\}$ is a disjoint collection of points of $T$ with $x_{i} \in T-\left\{t_{0}\right\}$, $1 \leqq i \leqq p$. The map $f$ is said to be transverse with respect to $\left\{x_{1}, \ldots, x_{p}\right\}$ if $f$ is transverse with respect to each $x_{i}$. In what follows the collection of points $\left\{x_{1}, \ldots, x_{p}\right\}$ will always be chosen so that no two members belong to the same simple closed curve $T_{i}$ of the wedge $T$. Notice that if $f$ is transverse with respect to $\left\{x_{1}, \ldots, x_{p}\right\}$, then the product structure for each component of $f^{-1}\left(U\left(x_{i}\right)\right)$ is defined so that $f$ maps the positive side, $U^{+}(J)$ (negative side, $U^{-}(J)$ ), of $J$ to the positive side (negative side) of $x_{i}$ in $U\left(x_{i}\right)$.

Suppose $f$ is a PL map of $\left(S, s_{0}\right)$ into $\left(T, t_{0}\right)$ and for some collection of points $\left\{x_{1}, \ldots, x_{p}\right\}$ in $T-\left\{t_{0}\right\}$ the map $f$ is transverse with respect to $\left\{x_{1}, \ldots, x_{p}\right\}$. Each component of $f^{-1}\left(x_{i}\right)$ is a two-sided polyhedral simple closed curve in $S$. Suppose $J_{0}$ and $J_{1}$ are distinct components of $f^{-1}\left(x_{j}\right)$ for a fixed $j$. Suppose that $A$ is a polyhedral arc in $S-\left\{s_{0}\right\}$ from $J_{0}$ to $J_{1}$ such that $A \cap \bigcup_{i=1}^{p} f^{-1}\left(x_{i}\right)=\operatorname{Bd} A$.

Let $Q_{0} \subset J_{0}$ and $Q_{1} \subset J_{1}$ be small arcs each containing an endpoint of $A$ in its interior. Suppose $Q \subset S-\left\{s_{0}\right\}$ is a regular neighborhood of $A$ so that $A$ is a spanning arc of $Q$, and $Q$ meets $\bigcup_{i=1}^{p} f^{-1}\left(x_{i}\right)$ only in $Q_{0} \cup Q_{1} \subset \mathrm{Bd} Q$. Then the closure of Bd $Q-\left(Q_{0} \cup Q_{1}\right)$ consists of two disjoint arcs $A_{0}$ and $A_{1}$ from $J_{0}$ to $J_{1}$.

LEMMA 3.1. If $f$ maps the arc $A$ into the trivial loop of $T$ based at $f(\mathrm{Bd} A)$, then there is a PL map $g$ of $S$ into $T$ so that

(i) $g$ is homotopic to $f\left(\operatorname{Rel}\left\{s_{0}\right\}\right)$,

(ii) $g^{-1}\left(x_{i}\right)=f^{-1}\left(x_{i}\right), i \neq j$,

(iii) $g^{-1}\left(x_{j}\right)=f^{-1}\left(x_{j}\right) \cup\left(A_{0} \cup A_{1}\right)-\left(Q_{0} \cup Q_{1}\right)$, and

(iv) $g$ is transverse with respect to $\left\{x_{1}, \ldots, x_{p}\right\}$.

Proof. The proof of this lemma is straightforward with special care being given to the technical requirement that the resulting PL map $g$ be transverse with respect 
to $\left\{x_{1}, \ldots, x_{p}\right\}$. The PL map $g$ is constructed in two steps. In the first step, a PL map $h$ is found so that $h$ is homotopic to $f\left(\operatorname{Rel}\left\{s_{0}\right\}\right), h^{-1}\left(x_{i}\right)=f^{-1}\left(x_{i}\right), i \neq j$, and $h^{-1}\left(x_{j}\right)=f^{-1}\left(x_{j}\right) \cup A$. (Note that $h$ cannot be transverse with respect to $\left\{x_{1}, \ldots, x_{p}\right\}$ since one component of $h^{-1}\left(x_{j}\right)$ is not a simple closed curve.) The desired PL map $g$ is then obtained from $h$. The construction uses the fact that $\pi_{2}(T)=0$ to form the desired homotopies. Furthermore, the construction may be done so that $g$ does not differ from $h$ outside a small regular neighborhood of $Q$. Details of similar constructions appear in [3].

Let $S$ denote a closed surface and let $T$ denote a wedge at $t_{0}$ of $k$ simple closed curves $T_{1}, \ldots, T_{k}$. For $1 \leqq i \leqq k$, choose a point $t_{i} \in T_{i}-\left\{t_{0}\right\}$. Suppose $h$ is a map of $\left(S, s_{0}\right)$ to $\left(T, t_{0}\right)$ which is transverse with respect to $\bigcup_{i=1}^{k}\left\{t_{i}\right\}$. For each $i>0$ and in each component of $h^{-1}\left(t_{i}\right)$ choose a distinguished point. The collection of distinguished points for $h$ will be denoted $D_{h}$.

Let $\gamma$ be a polyhedral path in $S$ with its endpoints in $D_{h}$ and in general position with respect to $\bigcup_{i=1}^{k} h^{-1}\left(t_{i}\right)$. (The endpoints of $\gamma$ are not necessarily distinct.) The path $\gamma$ may be written as a product of paths

$$
\gamma=\gamma_{m}^{\prime} \cdots \gamma_{1}^{\prime},
$$

reading from right to left, where each $\gamma_{r}^{\prime}$ is a path in $S$ meeting $\bigcup_{i=1}^{k} h^{-1}\left(t_{i}\right)$ in precisely its endpoints. The orientation of the path $\gamma$ induces an orientation on each $\gamma_{r}^{\prime}, 1 \leqq r \leqq m$. This induced orientation is used when referring to the initial point and the terminal point of $\gamma_{r}^{\prime}$.

Let $K_{r}$ denote the component of $\bigcup_{i=1}^{k} h^{-1}\left(t_{i}\right)$ containing the terminal point of $\gamma_{r}^{\prime}$ and let $d_{r}$ denote $K_{r} \cap D_{h}$. Let $d_{0}$ denote the initial point of $\gamma$ and let $K_{0}$ denote the component of $\bigcup_{i=1}^{k} h^{-1}\left(t_{i}\right)$ containing $d_{0}$. If $U\left(K_{r}\right)$ is the neighborhood of $K_{r}$ chosen in part 2 of the definition for $h$ to be transverse with respect to $\bigcup_{i=1}^{k}\left\{t_{i}\right\}$, then for $1 \leqq r<m$ the paths $\gamma_{r}^{\prime}$ and $\gamma_{r+1}^{\prime}$ approach $K_{r}$ from different sides of $U\left(K_{r}\right)$. The notation $U^{r}\left(K_{r}\right), U^{r+1}\left(K_{r}\right)$ will be used to denote the side of $K_{r}$ in $U\left(K_{r}\right)$ that $\gamma_{r}^{\prime}, \gamma_{r+1}^{\prime}$ approaches, respectively.

Let $\mu_{r}$ be an arc in $K_{r}$ from $d_{r}$ to the terminal point of $\gamma_{r}^{\prime}$ ( $\mu_{r}$ is not unique). The path $\gamma$ is homotopic $\left(\operatorname{Rel}\left\{d_{0} \cup d_{m}\right\}\right)$ to the path

$$
\left(\gamma_{m}^{\prime} \mu_{m-1}\right) \cdots\left(\mu_{2}^{-1} \gamma_{2}^{\prime} \mu_{1}\right)\left(\mu_{1}^{-1} \gamma_{1}^{\prime}\right)
$$

reading from right to left. Define $\mu_{0}$ and $\mu_{m}$ to be the constant paths at $d_{0}$ and $d_{m}$ respectively. Then

$$
\gamma=\gamma_{m} \cdots \gamma_{1}
$$

where $\gamma_{r}=\mu_{r}^{-1} \gamma_{r}^{\prime} \mu_{r-1}, 1 \leqq r \leqq m$, is called a canonical form for $\gamma$ relative to $h$. Notice that for each $r$, the path $h / \gamma_{r}$ is homotopic $\left(\operatorname{Rel}\left\{h\left(d_{r-1}\right) \cup h\left(d_{r}\right)\right\}\right)$ to $h / \gamma_{r}^{\prime}$.

Let $S^{1}$ denote the unit circle with a fixed orientation and let $x_{0}$ denote a base point for $S^{1}$. For $1 \leqq i \leqq k$, let $U\left(t_{i}\right)$ denote the intervals obtained about $t_{i}$ in part 2 of the definition for $h$ to be transverse with respect to $\bigcup_{i=1}^{k}\left\{t_{i}\right\}$. Let $\bar{t}_{i}^{\prime}$ be a PL homeomorphism of $\left(S^{1}, x_{0}\right)$ onto $\left(T_{i}, t_{0}\right)$ so that the orientation induced on the 
loop $\bar{t}_{i}^{\prime}$ agrees with that of $U\left(t_{i}\right)$; i.e. when traveling along $\bar{t}_{i}^{\prime}$ in a positive direction the negative side of $t_{i}$ in $U\left(t_{i}\right)$ is met prior to the positive side of $t_{i}$ in $U\left(t_{i}\right)$. Let $a_{j}$ be an arc from $t_{j}$ to $t_{0}$ in $T_{j}$ for some fixed $j$. The loops $\bar{t}_{i}=a_{j}^{-1} \bar{t}_{i}^{\prime} a_{j}, 1 \leqq i \leqq k$, form a collection of free generators for the free group $\pi_{1}\left(T, t_{j}\right)$.

If $\gamma$ is a path in $S$ with its endpoints in $h^{-1}\left(t_{j}\right) \cap D_{h}$ and $\gamma=\gamma_{m} \cdots \gamma_{1}$ is a canonical form for $\gamma$ relative to $h$, then there is a procedure for geometrically deciding the word in the symbols $\bar{t}_{1}, \bar{t}_{1}^{-1}, \ldots, \bar{t}_{k}, \bar{t}_{k}^{-1}$ that corresponds to the loop $h / \gamma$ in $\pi_{1}\left(T, t_{j}\right)$. The procedure used throughout this section is described in the next two paragraphs.

For $1 \leqq r<m$, there is a single symbol from the collection $\left\{\bar{t}_{1}, \bar{t}_{1}^{-1}, \ldots, \bar{t}_{k}, \bar{t}_{k}^{-1}\right\}$ corresponding to $d_{r}$. If $h\left(d_{r}\right)=t_{i}$, then corresponding to $d_{r}$ is the symbol $\bar{t}_{i}$ or $\bar{t}_{i}^{-1}$ according to $U^{r}\left(K_{r}\right)=\bar{U}\left(K_{r}\right)$ or $U^{r}\left(K_{r}\right)=U^{+}\left(K_{r}\right)$, respectively. The corresponding of a symbol to $d_{0}$ and a symbol to $d_{m}$ must be considered simultaneously. By the choices of $\gamma$, it follows that $h\left(d_{0}\right)=h\left(d_{m}\right)=t_{j}$. There are two cases:

Case (1). Corresponding to $d_{0}$ is no symbol and corresponding to $d_{m}$ is the symbol $\bar{t}_{j}\left(\bar{t}_{j}^{-1}\right)$, if $\gamma_{1}^{\prime}$ approaches $K_{0}$ from $U^{+}\left(K_{0}\right)\left(U^{-}\left(K_{0}\right)\right)$ and $\gamma_{m}^{\prime}$ approaches $K_{m}$ from $U^{-}\left(K_{m}\right)\left(U^{+}\left(K_{m}\right)\right)$.

Case (2). Corresponding to $d_{0}$ is the symbol $\bar{t}_{j}\left(\bar{t}_{j}^{-1}\right)$ and corresponding to $d_{m}$ is the symbol $\bar{t}_{j}^{-1}\left(\bar{t}_{j}\right)$ if $\gamma_{1}^{\prime}$ approaches $K_{0}$ from $U^{+}\left(K_{0}\right)\left(U^{-}\left(K_{0}\right)\right)$ and $\gamma_{m}^{\prime}$ approaches $K_{m}$ from $U^{+}\left(K_{m}\right)\left(U^{-}\left(K_{m}\right)\right)$.

It is clear that this procedure does indeed give a word $w$ in the symbols $\left\{\bar{t}_{1}, \bar{t}_{1}^{-1}, \ldots, \bar{t}_{k}, \bar{t}_{k}^{-1}\right\}$. Furthermore, the word $w$ is either of length $m$ or $m+1$ depending on Case (1) or Case (2) respectively. That the word $w$ determines a representative of the class of $h / \gamma$ in $\pi_{1}\left(T, t_{j}\right)$ with generators $\bar{t}_{1}, \ldots, \bar{t}_{k}$ follows from $\gamma$ being in canonical form relative to $h$.

LemMa 3.2. Let $S$ be a closed surface. Suppose $f$ is a map of $S$ into $T$, a wedge at $t_{0}$ of $k$ simple closed curves $T_{1}, \ldots, T_{k}$, and $f\left(s_{0}\right)=t_{0}$. If $f_{*}$ is an epimorphism, then there is a PL map $g$ of $S$ into $T$ so that

(i) $\mathrm{g}$ is homotopic to $f\left(\operatorname{Rel}\left\{s_{0}\right\}\right)$;

(ii) for each $i, 1 \leqq i \leqq k$, there is a point $t_{i} \in T_{i}-\left\{t_{0}\right\}$ so that $g^{-1}\left(t_{i}\right)$ is a single simple closed curve $J_{i}$ in $S$ and $g$ is transverse with respect to $\left\{t_{1}, \ldots, t_{k}\right\}$; and

(iii) $S-\bigcup_{i=1}^{k} J_{i}$ is connected.

Proof. To begin, the map $f$ may be taken as a PL map with $f\left(s_{0}\right)=t_{0}$ [2], [15]. Let $t_{i} \in T_{i}-\left\{t_{0}\right\}$ be a nonvertex point of a subdivision of $T$ for which $f$ is simplicial. Then the components of $f^{-1}\left(t_{i}\right)$ are two-sided polyhedral simple closed curves in $S, 1 \leqq i \leqq k$, and $f$ is transverse with respect to $\bigcup_{i=1}^{k}\left\{t_{i}\right\}$. The proof of the lemma is accomplished by "surgery" on PL maps in the same homotopy class as $f\left(\operatorname{Rel}\left\{s_{0}\right\}\right)$.

Consider the collection $\mathscr{F}$ of PL maps of $\left(S, s_{0}\right)$ to $\left(T, t_{0}\right)$ which are homotopic to $f\left(\operatorname{Rel}\left\{s_{0}\right\}\right)$ and which are transverse with respect to $\bigcup_{i=1}^{k}\left\{t_{i}\right\}$. For $h \in \mathscr{F}$, let $c(h, i)$ be one less than the number of components of $h^{-1}\left(t_{i}\right)$. Since $f_{*}$ is an epimorphism and since a member of $\mathscr{F}$ is homotopic to $f\left(\operatorname{Rel}\left\{s_{0}\right\}\right)$ then, $c(h, i) \geqq 0$ for each $h \in \mathscr{F}$ and each $i, 1 \leqq i \leqq k$. Hence, with this notation it is desired to show 
that there is a member $g$ of $\mathscr{F}$ so that $\sum_{i=1}^{k} c(g, i)=0$. In order to obtain such a member $g$ of $\mathscr{F}$, it will be shown that for any member $h$ of $\mathscr{F}$ for which $\sum_{i=1}^{k} c(h, i)$ $=n>0$, there is a member $h^{\prime}$ of $\mathscr{F}$ with $\sum_{1}^{k} c\left(h^{\prime}, i\right)=n-1$.

Suppose $h \in \mathscr{F}$ and $\sum_{1}^{k} c(h, i)=n>0$. Then $c(h, i)>0$ for some $i, 1 \leqq i \leqq k$. Thus $h^{-1}\left(t_{i}\right)$ has at least two components. Let $L_{0}$ and $L_{1}$ denote distinct components of $h^{-1}\left(t_{i}\right)$. Let $D_{h}$ denote the collection of distinguished points of $h$ and let $d_{0}=L_{0} \cap D_{h}$ while $d=L_{1} \cap D_{h}$. Choose a polyhedral path $\alpha$ in $S$ from $d_{0}$ to $d$. Since $h_{*}$ is an epimorphism and $h / \alpha$ is a loop in $\pi_{1}\left(T, t_{i}\right)$, there is a loop $\beta$ in $S$ based at $d$ so that if $\beta \alpha^{-1}$ is the product of the path $\alpha^{-1}$ with $\beta$, then the loop $h / \beta \alpha^{-1}$ is trivial in $\pi_{1}\left(T, t_{i}\right)$. Let

$$
\gamma=\gamma_{m} \cdots \gamma_{1}
$$

be a canonical form for the path $\gamma=\beta \alpha^{-1}$ relative to $h$ in $S$. Note that $\gamma_{1}$ has an endpoint at $d_{0}$ in $L_{0}$ and $\gamma_{m}$ has an endpoint at $d_{m}=d$ in $L_{1}$.

Since $\gamma$ is in canonical form relative to $h$ and both endpoints of $\gamma$ are in $D_{h} \cap h^{-1}\left(t_{i}\right)$ for a fixed $i$, the loop $h / \gamma$ may be written as a word $w$ in the symbols $\bar{t}_{1}, \bar{t}_{1}^{-1}, \ldots, \bar{t}_{k}, \bar{t}_{k}^{-1}$ as described above. The word $w$ is equivalent to 1 in $\pi_{1}\left(T, t_{i}\right)$ and $w$ is nonvoid [4]. Hence, there is a cancellation of the form $\bar{t}_{j} \bar{t}_{j}^{-1}$ or $\bar{t}_{j}^{-1} \bar{t}_{j}$ for some $j, 1 \leqq j \leqq k$. There are two cases to consider.

Case (a). The cancellation $\bar{t}_{j} \bar{t}_{j}^{-1}$ or $\bar{t}_{j}^{-1} \bar{t}_{j}$ corresponds to $d_{r-1}$ and $d_{r}$ for some $r$, $1 \leqq r \leqq m$, where $d_{r-1} \neq d_{r}$. In this case, the path $\gamma_{r}$ connects distinct components of $h^{-1}\left(t_{j}\right)$ and the loop $h / \gamma_{r}$ is trivial in $\pi_{1}\left(T, t_{j}\right)$. By an earlier remark, it follows that the loop $h / \gamma_{r}^{\prime}$ is trivial in $\pi_{1}\left(T, t_{j}\right)$. Let $A$ be an arc in $\gamma_{r}^{\prime}$ from one endpoint of $\gamma_{r}^{\prime}$ to the other endpoint of $\gamma_{r}^{\prime}$. Then $A$ and $h$ satisfy the hypothesis of Lemma 3.1. If $h^{\prime}$ denotes the PL map guaranteed in the conclusion of Lemma 3.1, then $c\left(h^{\prime}, i\right)=c(h, i), i \neq j$, and $c\left(h^{\prime}, j\right)=c(h, j)-1$. Furthermore, $h^{\prime} \in \mathscr{F}$.

Case (b). The cancellation $\bar{t}_{j} \bar{t}_{j}^{-1}$ or $\bar{t}_{j}^{-1} \bar{t}_{j}$ corresponds to $d_{r-1}$ and $d_{r}$ for some $r$, $1 \leqq r \leqq m$, where $d_{r-1}=d_{r}$. In this case, the path $\gamma_{r}$ is closed. Both paths $\gamma_{r+1}^{\prime}$ and $\gamma_{r-1}^{\prime}$ approach $K_{r}$ from the same side in $U\left(K_{r}\right)$, namely, $U^{r+1}\left(K_{r}\right)$.

Recall the properties of the path $\gamma$ :

(i) $\gamma$ is a path from $d_{0}$ to $d_{m}$ where $d_{0} \neq d_{m}$,

(ii) $\gamma$ has a canonical form relative to $h$,

$$
\gamma=\gamma_{m} \cdots \gamma_{1}
$$

(the length of such a canonical form relative to $h$ is" the integer $m$ ), and

(iii) If $w$ is the word in the symbols $\left\{\bar{t}_{1}, \bar{t}_{1}^{-1}, \ldots, \bar{t}_{k}, \bar{t}_{k}^{-1}\right\}$ which by the above procedure corresponds to the class of $h / \gamma$ in $\pi_{1}\left(T, t_{i}\right)$, then $w$ is equivalent to 1 . It will be shown that there is a path $\gamma^{\prime}$ so that

(i) $\gamma^{\prime}$ is a path from $d_{0}$ to $d_{m}, d_{0} \neq d_{m}$,

(ii) $\gamma^{\prime}$ has a canonical form relative to $h$,

$$
\gamma^{\prime}=\delta_{m^{\prime}} \cdots \delta_{1}
$$

where $m^{\prime}<m$, and 
(iii) if $w^{\prime}$ is the word in the symbols $\left\{t_{1}, t_{1}^{-1}, \ldots, t_{k}, t_{k}^{-1}\right\}$ which by the above procedure corresponds to the class of $h / \gamma^{\prime}$ in $\pi_{1}\left(T, t_{i}\right)$, then $w^{\prime}$ is equivalent to 1 .

The notation requires that the three situations $r=1,1<r<m$, and $r=m$ be considered separately.

Suppose that $r=1$. Then the path $\gamma^{\prime}$ and a desired canonical form for $\gamma^{\prime}$ relative to $h$ is

$$
\gamma^{\prime}=\gamma_{m} \cdots \gamma_{3} \delta
$$

where $\delta$ is obtained from the path $\left(\mu_{2}^{-1} \gamma_{2}^{\prime} \mu_{1}\right)$ by a small displacement of the arc $\mu_{1}$, except for the endpoint $d_{1}$, into $U^{2}\left(K_{1}\right)-K_{1}$.

Suppose that $1<r<m$. Then the path $\gamma^{\prime}$ and a desired canonical form for $\gamma^{\prime}$ relative to $h$ is

$$
\gamma^{\prime}=\gamma_{m} \cdots \gamma_{r+2} \delta \gamma_{r-2} \cdots \gamma_{1}
$$

where $\delta$ is obtained from the path

$$
\left(\mu_{r+1}^{-1} \gamma_{r+1}^{\prime} \mu_{r} \mu_{r-1}^{-1} \gamma_{r-1}^{\prime} \mu_{r-2}\right)
$$

by a small displacement of the path $\mu_{r} \mu_{r-1}^{-1}$ into $U^{r+1}\left(K_{r}\right)-K_{r}$.

Suppose $r=m$. Then the path $\gamma^{\prime}$ and a desired canonical form for $\gamma^{\prime}$ relative to $h$ is

$$
\gamma^{\prime}=\delta \gamma_{m-2} \cdots \gamma_{1}
$$

where $\delta$ is obtained from the path $\mu_{m-1}^{-1} \gamma_{m-1}^{\prime} \mu_{m-2}$ by a small displacement of the arc $\mu_{m-1}$, except for the endpoint $d_{m-1}$ into $U^{m-1}\left(K_{m-1}\right)-K_{m-1}$.

Since the length of a canonical form for the path $\gamma^{\prime}$ in any of the above situations is of length less than $m$ and $d_{0} \neq d_{m}$, there is, after a finite number of applications of Case (b), a path from $d_{0}$ to $d_{m}$ and a canonical form for this path relative to $h$ so that Case (a) is satisfied.

To complete the proof of Lemma 4.1, it is necessary to show that if $g$ is a member of $\mathscr{F}$ so that $\sum_{i=1}^{k} c(g, i)=0$ and if $J_{i}=g^{-1}\left(t_{i}\right)$, then $S-\bigcup_{i=1}^{k} J_{i}$ is connected. This is done by showing that there is a collection of loops $l_{1}, \ldots, l_{k}$ based at $s_{0}$ in $S$ so that $l_{i} \cap J_{i}$ is a single point where $l_{i}$ crosses $J_{i}$ and $l_{i} \cap J_{j}=\varnothing, i \neq j$.

The PL map $g$ induces a homomorphism $g_{*}$ of $\pi_{1}\left(S, s_{0}\right)$ onto $\pi_{1}\left(T, t_{0}\right)$. Let $\bar{t}_{1}^{\prime}, \ldots, \bar{t}_{k}^{\prime}$ be the generators of the free group $\pi_{1}\left(T, t_{0}\right)$ described earlier. Let $\gamma_{i}$ be a loop in $\pi_{1}\left(S, s_{0}\right)$ so that $g_{*}\left(\gamma_{i}\right)$ is equivalent to $\bar{t}_{i}^{\prime}$ as elements of $\pi_{1}\left(T, t_{0}\right)$ and $\gamma_{i}$ is in canonical form relative to $g$. The endpoints of $\gamma_{i}$ are both at $s_{0}$ and not in $D_{g}$; hence, the canonical form is slightly altered from that above. To obtain a form analogous to that above, add the point $s_{0}$ to the collection of distinguished points $D_{g}$.

In the remainder of the argument, assume $i$ is fixed. Since there is no concern for how $l_{i}$ meets $l_{j}, i \neq j$, the loops $l_{i}$ may be chosen one at a time. Let

$$
\gamma_{i}=\gamma_{i m} \cdots \gamma_{i 1}
$$

be a canonical form for $\gamma_{i}$ relative to $g$. Note that $\gamma_{i 1}$ and $\gamma_{i m}$ each have an endpoint at $s_{0}$ and $m \geqq 2$. 
By the above procedure, it is possible to choose a word $w_{i}$ of $\pi_{1}\left(T, t_{0}\right)$ in the symbols $\left\{\bar{t}_{1}^{\prime}, \bar{t}_{1}^{\prime-1}, \ldots, \bar{t}_{k}^{\prime}, \bar{t}_{k}^{\prime-1}\right\}$ corresponding to the loop $g / \gamma_{i}$ based at $t_{0}$. By the choice of $\gamma_{i}, w_{i}$ is equivalent to $\bar{t}_{i}^{\prime}$. In order to obtain the desired loop $l_{i}$ from $\gamma_{i}$, an inductive argument on the length $m$ of the given canonical form for $\gamma_{i}$ relative to $g$ is used.

If $m=2$, then

$$
\gamma_{i}=\gamma_{i 2} \gamma_{i 1}=\left(\mu_{2}^{-1} \gamma_{i 2}^{\prime} \mu_{1}\right)\left(\mu_{1}^{-1} \gamma_{i 1}^{\prime} \mu_{0}\right)
$$

where $\mu_{0}$ and $\mu_{2}$ are constant paths at $s_{0}$ and $\mu_{1}$ is an arc in $J_{i}$ from the distinguished point of $J_{i}$ to the terminal point of $\gamma_{i 1}^{\prime}$. Let $l_{i}=\gamma_{i 2}^{\prime} \gamma_{i 1}^{\prime}$.

Hence it is assumed that if $\gamma_{i}$ is a loop based at $s_{0}$ with $g_{*}\left(\gamma_{i}\right)$ equivalent to $\bar{t}_{i}^{\prime}$ as elements in $\pi_{1}\left(T, t_{0}\right)$ and if $\gamma_{i}$ has a canonical form relative to $g$ of length less than $m$, where $m>2$, then the desired loop $l_{i}$ based at $s_{0}$ may be obtained. Let

$$
\gamma_{i}=\gamma_{i m} \cdots \gamma_{i 1}
$$

be a canonical form for $\gamma_{i}$ relative to $g$ where $m>2$. Since $w_{i}$ is equivalent to $\bar{t}_{i}^{\prime}$ and $m-1 \geqq 2$, there is a cancellation of the form $\bar{t}_{j}^{\prime} \bar{t}_{j}^{\prime-1}$ or $\bar{t}_{j}^{\prime-1} \bar{t}_{j}^{\prime}$ in $w_{i}$. Suppose this cancellation corresponds to $d_{r-1}$ and $d_{r}$ for some $r, 1<r<m$. Then the path $\gamma_{i r}$ is a closed loop and $d_{r-1}=d_{r}$. This is true since $g^{-1}\left(t_{j}\right)=J_{j}$ is a single simple closed curve. Let $\delta_{r}^{\prime}$ be the path from $d_{r-2}$ to $d_{r+1}$ defined as

$$
\delta_{r}^{\prime}=\left(\mu_{r+1}^{-1} \gamma_{i(r+1)}^{\prime} \mu_{r}\right)\left(\mu_{r-1}^{-1} \gamma_{i(r-1)}^{\prime} \mu_{r-2}\right) .
$$

Since $\gamma_{i(r+1)}^{\prime}$ and $\gamma_{i(r-1)}^{\prime}$ approach $K_{r}=J_{j}$ from the same side, a small displacement of $\left(\mu_{r} \mu_{r-1}^{-1}\right)$ into $U^{r+1}\left(K_{r}\right)-K_{r}$ determines a path $\delta_{r}$ from $d_{r-2}$ to $d_{r+1}$ so that $\delta_{r}$ is homotopic in $S$ to $\delta_{r}^{\prime}\left(\operatorname{Rel}\left\{d_{r-2} \cup d_{r+1}\right\}\right)$ and the path

$$
\gamma_{i}^{\prime}=\gamma_{i m} \cdots \gamma_{i(r+2)} \delta_{r} \gamma_{i(r-2)} \cdots \gamma_{i 1}
$$

is in canonical form relative to $h$. The paths $\gamma_{i(r+2)}$ or $\gamma_{i(r-2)}$ are constant paths at $s_{0}$ if $r=m-1$ or $r=2$, respectively. The length of this canonical form for $\gamma_{i}^{\prime}$ is less than $m$ and $h / \gamma_{i}^{\prime}=\bar{t}_{i}^{\prime}$. Hence, applying the inductive hypothesis, the desired loop $l_{i}$ may be obtained from $\gamma_{i}^{\prime}$.

This completes the proof of Lemma 3.2.

REMARK. The technique for finding the arc $A$ in the proof of Lemma 3.2 is suggested by the technique introduced in [10] by J. Stallings to find a "binding tie".

The following corollary dealing with homomorphisms of the fundamental group of a closed surface $S$ onto a free group is a direct consequence of the preceding lemma.

COROLlaRY 3.3. Let $S$ be a closed surface with Euler characteristic $2-p, p \geqq 0$. Then there is a homomorphism of $\pi_{1}(S)$ onto a free group of rank $k$ if and only if $k \leqq[p / 2]$, where $[p / 2]$ is the greatest integer $\leqq p / 2$. 


\section{Handlebodies as mapping cylinders.}

LEMMA 4.1. Let $S$ be a closed surface with $\chi(S)=2-2 n$ for $n>0$. Suppose $f$ is a map of $S$ into $T$, a wedge of $n$ simple closed curves at $t_{0}$, and $f\left(s_{0}\right)=t_{0}$. If $f_{*}$ is an epimorphism, then there is a PL map $g$ of $S$ into $T$ so that

(i) $g$ is homotopic to $f\left(\operatorname{Rel}\left\{s_{0}\right\}\right)$ and

(ii) the mapping cylinder of $g$ is topologically equivalent to a handlebody (possible nonorientable) of genus $n$.

Proof. The map $f$ satisfies the hypothesis of Lemma 3.2. Let $h$ denote the PL map guaranteed in the conclusion of that lemma. For each $i, 1 \leqq i \leqq n$, let $U\left(t_{i}\right)$ be an interval in $T$ with $t_{i}$ in its interior and let $U\left(J_{i}\right)$ be a component of $h^{-1}\left(U\left(t_{i}\right)\right)$ which is a product neighborhood of $J_{i}=h^{-1}\left(t_{i}\right)$ in $S$ so that $U\left(t_{i}\right)$ and $U\left(J_{i}\right)$ satisfy part 2 of the definition for $h$ to be transverse with respect to $\left\{t_{1}, \ldots, t_{n}\right\}$.

There is a homotopy $h_{p}, 0 \leqq p \leqq 1$, of $T$ onto $T$ so that for each $p$ and each integer $i, 0 \leqq i \leqq n, h_{p}\left(t_{i}\right)=t_{i}, h_{0}=$ identity on $T, h_{1}$ is $\mathrm{PL}, h_{1}\left(T-\bigcup_{i=1}^{n} U^{\circ}\left(t_{i}\right)\right)=t_{0}$, and $h_{1} / U^{\circ}\left(t_{i}\right)$ is a homeomorphism of $U^{\circ}\left(t_{i}\right)$ onto $T_{i}-t_{0}$. Let $g$ be the PL map defined as $g=h_{1} \circ h$. The homotopy from $h$ to $g\left(\operatorname{Rel}\left\{s_{0}\right\}\right)$ is $h_{p} \circ h, 0 \leqq p \leqq 1$.

Let $\phi_{i}$ denote the homeomorphism from $t_{i} \times[-1,1]$ onto $U\left(t_{i}\right)$ and let $\psi_{i}$ be the homeomorphism of $J_{i} \times[-1,1]$ onto $U\left(J_{i}\right)$ corresponding to $\phi_{i}$ and satisfying part 2 of the definition for $h$ to be transverse with respect to $\left\{t_{1}, \ldots, t_{n}\right\}$. Define $\phi_{i}^{\prime}$ to be the PL map $h_{1} \phi_{i}$. Then for $-1<r<1$,

and

$$
g \psi_{i}\left(J_{i} \times r\right)=\phi_{i}^{\prime}\left(t_{i} \times r\right)
$$

$$
g\left(S-\bigcup_{i=1}^{n} U^{\circ}\left(J_{i}\right)\right)=t_{0}
$$

It follows that $g \psi_{i}\left(J_{i} \times(-1)\right)=t_{0}=g \psi_{i}\left(J_{i} \times 1\right)$.

It will be shown that the mapping cylinder, $C(g)$, of $g$ is homeomorphic to a handlebody of genus $n$.

Let $B$ be the closed unit ball in $E^{3}$. Choose $2 n$ distinct points on $\operatorname{Bd} B ; p_{1}, q_{1}, \ldots$, $p_{n}, q_{n}$. Let $\bar{p}_{i}, \bar{q}_{i}$ denote the straight line segment in $B$ from the origin to $p_{i}, q_{i}$ respectively, $1 \leqq i \leqq n$. Choose $2 n$ mutually exclusive spherical 2 -simplexes on $\operatorname{Bd} B, \Delta\left(p_{1}\right), \Delta\left(q_{1}\right), \ldots, \Delta\left(p_{n}\right), \Delta\left(q_{n}\right)$, where $p_{i} \in \Delta^{\circ}\left(p_{i}\right)$ and $q_{i} \in \Delta^{\circ}\left(q_{i}\right)$. Let $\bar{\Delta}\left(p_{i}\right)$, $\bar{\Delta}\left(q_{i}\right)$ denote the cones from the origin over $\Delta\left(p_{i}\right), \Delta\left(q_{i}\right)$ respectively.

The mapping cylinder, $C(g)$, of $g$ is obtained from the disjoint union of $S \times I$ and $T$ by identifying each $s \times 1$ with $g(s)$. Using the above notation, let $V\left(J_{i}\right)=$ $\psi_{i}\left(J_{i} \times[-1 / 2,1 / 2]\right)$ which is contained in $U^{\circ}\left(J_{i}\right)$. Let

$$
S^{\prime}=S-\bigcup_{i=1}^{n} V^{\circ}\left(J_{i}\right)
$$

a sphere with $2 n$ holes. Let $V\left(t_{i}\right)=g\left(V\left(J_{i}\right)\right)$ and let

$$
T^{\prime}=T-\bigcup_{i=1}^{n} V^{\circ}\left(t_{i}\right)
$$


Consider the subset of $C(g)$ obtained from the disjoint union of $S^{\prime} \times I$ and $T^{\prime}$ by identifying $s^{\prime} \times 1$ with $g\left(s^{\prime}\right)$ for each $s^{\prime} \in S^{\prime}$. There is a homeomorphism $G$ of this space onto $B$ so that

(a) $G\left(T^{\prime}\right)=\bigcup_{i=1}^{n} \bar{p}_{i} \cup \bigcup_{i=1}^{n} \bar{q}_{i}$,

(b) $G\left(t_{0}\right)$ is the origin, and

(c) for every $i, 1 \leqq i \leqq n$, the subset obtained from the disjoint union of $\left(U\left(J_{i}\right)-V^{\circ}\left(J_{i}\right)\right) \times I$ and $T_{i}-V^{\circ}\left(t_{i}\right)$ by identifying $u \times 1$ with $g(u)$ for each $u \in U\left(J_{i}\right)$ $-V^{\circ}\left(J_{i}\right)$ has two components $\bar{P}_{i}$ and $\bar{Q}_{i}$ and $G / \bar{P}_{i}$ is a homeomorphism onto $\bar{\Delta}\left(p_{i}\right)$ while $G / \bar{Q}_{i}$ is a homeomorphism onto $\bar{\Delta}\left(q_{i}\right)$. Furthermore, if the disks in this space which correspond to the two components of $\operatorname{Bd}\left(V\left(J_{i}\right)\right) \times I$ after identification are denoted $P_{i}$ and $Q_{i}$, then $G\left(P_{i}\right)$ is a subdisk of $\Delta^{\circ}\left(p_{i}\right)$ with $p_{i}$ in its interior and $G\left(Q_{i}\right)$ is a subdisk of $\Delta^{\circ}\left(q_{i}\right)$ with $q_{i}$ in its interior.

To complete the homeomorphism of $C(g)$ to the regular neighborhood of a finite, connected, graph of Euler characteristic $1-n$, each subset of $C(g)$ obtained from the disjoint union of $V\left(J_{i}\right) \times I$ and $V\left(t_{i}\right)$ by identifying $v \times 1$ with $g(v)$ is homeomorphic to a handle (possibly nonorientable) from the disk $G\left(P_{i}\right)$ in $\mathrm{Bd} B$ to the disk $G\left(Q_{i}\right)$ in $\mathrm{Bd} B$.

REMARK. A handlebody $H$ is orientable if and only if $\mathrm{Bd} H$ is orientable.

5. Constructing three-manifolds. Let $S$ be a closed, orientable surface of genus $n>0$. Let $F_{1}$ and $F_{2}$ denote free groups of rank $n$ and let $F_{1} \times F_{2}$ denote their direct product [4]. Fix a point $s_{0}$ of $S$ and suppose $\eta_{1}$ is a homomorphism of $\pi_{1}\left(S, s_{0}\right)$ onto $F_{1}$ and $\eta_{2}$ is a homomorphism of $\pi_{1}\left(S, s_{0}\right)$ onto $F_{2}$. The homomorphism

$$
\left(\eta_{1}, \eta_{2}\right): \pi_{1}\left(S, s_{0}\right) \rightarrow F_{1} \times F_{2}
$$

defined as $\left(\eta_{1}, \eta_{2}\right)(l)=\left(\eta_{1}(l), \eta_{2}(l)\right)$ is called a splitting homomorphism of $\pi_{1}\left(S, s_{0}\right)$. A splitting homomorphism

$$
\left(\mu_{1}, \mu_{2}\right): \pi_{1}\left(R, r_{0}\right) \rightarrow G_{1} \times G_{2}
$$

of $\pi_{1}\left(R, r_{0}\right)$ is said to be equivalent to $\left(\eta_{1}, \eta_{2}\right)$ if there are isomorphisms $\alpha$ of $\pi_{1}\left(S, s_{0}\right)$ onto $\pi_{1}\left(R, r_{0}\right), \alpha_{1}$ of $F_{1}$ onto $G_{1}$, and $\alpha_{2}$ of $F_{2}$ onto $G_{2}$ so that the diagram

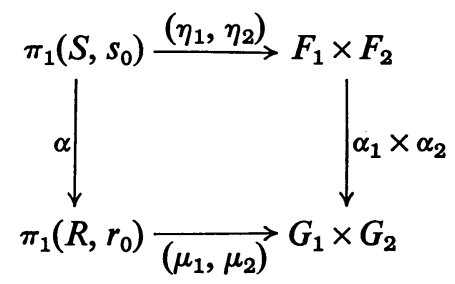

commutes. The homomorphism $\alpha_{1} \times \alpha_{2}$ is defined as

$$
\alpha_{1} \times \alpha_{2}\left(a_{1}, a_{2}\right)=\left(\alpha_{1} a_{1}, \alpha_{2} a_{2}\right) \text {. }
$$

Let $M$ be a closed, orientable three-manifold. It is known [8] that $M=U \cup V$ where $U$ and $V$ are cubes-with-handles and $U \cap V$ is their common boundary. 
The pair $(U, V)$ is called a Heegaard splitting of $M$. The genus of $(U, V)$ is defined to be the genus of $S=\mathrm{Bd} U=\mathrm{Bd} V$.

If $(P, Q)$ is a Heegaard splitting of the three-manifold $N$, then $(P, Q)$ is said to be equivalent to $(U, V)$ if and only if there is a homeomorphism $h$ of $M$ onto $N$ so that $h(U)=P$ and $h(V)=Q$.

Choose $s_{0} \in S$. The inclusion maps of $S$ into $U$ and into $V$ induce homomorphisms $u_{*}$ and $v_{*}$ of $\pi_{1}\left(S, s_{0}\right)$ onto $\pi_{1}\left(U, s_{0}\right)$ and onto $\pi_{1}\left(V, s_{0}\right)$, respectively. The splitting homomorphism

$$
\left(u_{*}, v_{*}\right): \pi_{1}\left(S, s_{0}\right) \rightarrow \pi_{1}\left(U, s_{0}\right) \times \pi_{1}\left(V, s_{0}\right)
$$

is called the splitting homomorphism of $\pi_{1}\left(S, s_{0}\right)$ induced by $(U, V)$. Suppose the Heegaard splittings $(U, V)$ of $M$ and $(P, Q)$ of $N$ are equivalent. Let $\left(u_{*}, v_{*}\right)$ and $\left(p_{*}, q_{*}\right)$ denote the splitting homomorphisms of $\pi_{1}(\mathrm{Bd} U)$ and $\pi_{1}(\mathrm{Bd} P)$ induced by $(U, V)$ and $(P, Q)$, respectively. Then $\left(u_{*}, v_{*}\right)$ and $\left(p_{*}, q_{*}\right)$ are equivalent splitting homomorphisms.

The following result was noticed by Stallings in [9]. A proof appears there.

LEMMA 5.1. Using the above notation, $\pi_{1}\left(M, s_{0}\right)$ is isomorphic to the quotient group of $\pi_{1}\left(S, s_{0}\right)$ by $\left(\right.$ kernel $\left.u_{*}\right) \cdot\left(\right.$ kernel $\left.v_{*}\right)$.

The next two theorems show that there is a one-one correspondence between equivalence classes of splitting homomorphisms and equivalence classes of Heegaard splittings.

THEOREM 5.2. Let $S$ be a closed, orientable surface of genus $n>0$. Suppose $\left(\eta_{1}, \eta_{2}\right)$ is a splitting homomorphism of $\pi_{1}\left(S, s_{0}\right)$ into $F_{1} \times F_{2}$. Then there is a closed, orientable 3-manifold $M$ and a Heegaard splitting $(U, V)$ of $M$ so that the splitting homomorphism induced by $(U, V)$ is equivalent to $\left(\eta_{1}, \eta_{2}\right)$.

Proof. For $k=1,2$ let $T^{k}$ be a wedge at $t_{0}^{k}$ of $n$ simple closed curves. Identify $\pi_{1}\left(T^{k}, t_{0}^{k}\right)$ with $F_{k}$. There is a map $f_{k}$ of $\left(S, s_{0}\right)$ into $\left(T^{k}, t_{0}^{k}\right)$ so that $\left(f_{k}\right)_{*}=\eta_{k}$. From Lemma 4.1 and $k=1,2$, there is a PL map $g_{k}$ of $\left(S, s_{0}\right)$ into $\left(T^{k}, t_{0}^{k}\right)$ so that $g_{k}$ is homotopic to $f_{k}\left(\operatorname{Rel}\left\{s_{0}\right\}\right)$ and the mapping cylinder $C\left(g_{k}\right)$ of $g_{k}$ is a cube-with-handles of genus $n$.

There is an isomorphism $\alpha_{k}$ of $\pi_{1}\left(C\left(g_{k}\right), s_{0}\right)$ onto $\pi_{1}\left(T^{k}, t_{0}^{k}\right)$ so that if $e_{k}$ is the inclusion of $S$ into $C\left(g_{k}\right)$, then $\alpha_{k}\left(e_{k}\right)_{*}=\left(f_{k}\right)_{*}$.

Let $M=C\left(g_{1}\right) \cup C\left(g_{2}\right)$ where $C\left(g_{1}\right) \cap C\left(g_{2}\right)=S$. Then $M$ is a closed, orientable 3-manifold. Furthermore, $\left(C\left(g_{1}\right), C\left(g_{2}\right)\right)$ is a Heegaard splitting of $M$ and $\left(\left(e_{1}\right)_{*},\left(e_{2}\right)_{*}\right)$ is the induced splitting homomorphism. The diagram

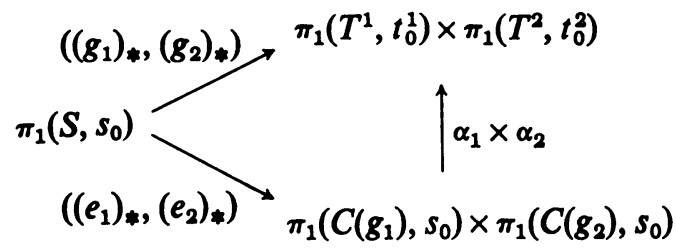

commutes. Since $\left(\left(g_{1}\right)_{*},\left(g_{2}\right)_{*}\right)=\left(\left(f_{1}\right)_{*},\left(f_{2}\right)_{*}\right)=\left(\eta_{1}, \eta_{2}\right)$, the theorem is proved. 
Theorem 5.3. Suppose $(U, V)$ is a Heegaard splitting of the three-manifold $M$ and $(P, Q)$ is a Heegaard splitting of the three-manifold $N$. If the splitting homomorphism induced by $(U, V)$ is equivalent to the splitting homomorphism induced by $(P, Q)$, then $(U, V)$ is equivalent to $(P, Q)$.

Proof. Let $S=\mathrm{Bd} U=\mathrm{Bd} V$ and $R=\mathrm{Bd} P=\mathrm{Bd} Q$. Choose $s_{0} \in S$ and $r_{0} \in R$. Let $\left(u_{*}, v_{*}\right)$ be the splitting homomorphism of $\pi_{1}\left(S, s_{0}\right)$ induced by $(U, V)$ and let $\left(p_{*}, q_{*}\right)$ be the splitting homomorphism of $\pi_{1}\left(R, r_{0}\right)$ induced by $(P, Q)$. Since $\left(u_{*}, v_{*}\right)$ is equivalent to $\left(p_{*}, q_{*}\right)$ there are isomorphisms $\alpha$ of $\pi_{1}\left(S, s_{0}\right)$ onto $\pi_{1}\left(R, r_{0}\right)$, $\alpha_{1}$ of $\pi_{1}\left(U, s_{0}\right)$ onto $\pi_{1}\left(P, r_{0}\right)$, and $\alpha_{2}$ of $\pi_{1}\left(V, s_{0}\right)$ onto $\pi_{1}\left(Q, r_{0}\right)$ so that the diagram

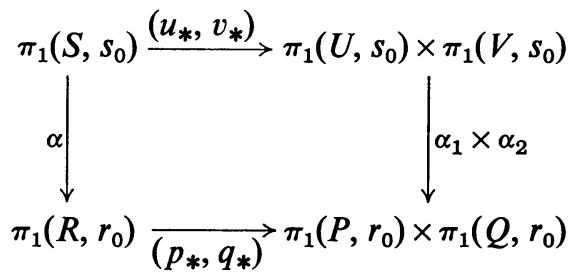

commutes.

There is a homeomorphism $h$ of $\left(S, s_{0}\right)$ onto $\left(R, r_{0}\right)$ so that $h_{*}=\alpha$ [5], [6]. The homeomorphism $h$ of $\left(S, s_{0}\right)$ onto $\left(R, r_{0}\right)$ can be extended to a homeomorphism of $U$ onto $P$ and $V$ onto $Q$ if and only if $h_{*}\left(\operatorname{kernel}\left(u_{*}\right)\right) \subset \operatorname{kernel}\left(p_{*}\right)$ and

$$
h_{*}\left(\operatorname{kernel}\left(v_{*}\right)\right) \subset \operatorname{kernel}\left(q_{*}\right)
$$

[7]. But this is true since $h_{*}=\alpha_{*}$ and the above diagram commutes.

6. An algebraic conjecture equivalent to Poincaré's Conjecture. In this section it is shown that two conjectures made by J. Stallings are equivalent to Poincaré's Conjecture that every simply connected, closed 3-manifold is topologically equivalent to the 3-sphere. One of these conjectures is algebraic in nature. A recent result by $R$. Traub [11] has shown that another purely algebraic conjecture is equivalent to Poincaré's Conjecture.

The following result of J. Stallings appears as Theorem 1 of [9].

LEMMA 6.1. Let $(U, V)$ be a Heegaard splitting of the closed, orientable 3-manifold $M$ where $\mathrm{Bd} U \doteq \mathrm{Bd} V=S$. Let $\left(u_{*}, v_{*}\right)$ be the splitting homomorphism of $\pi_{1}\left(S, s_{0}\right)$ induced by $(U, V)$. Then $\left(u_{*}, v_{*}\right)$ is an epimorphism if and only if $M$ is simply connected.

The notation $G_{1} * G_{2}$ is used for the free product of the groups $G_{1}$ and $G_{2}$ ([4, Vol. II, p. 11]). A homomorphism $\phi$ of a group $G$ into a free product $G_{1} * G_{2}$ is essential if there is no element $g \in G_{1} * G_{2}$ such that $g \phi(G) g^{-1}$ is contained in one of the factors $G_{1}$ or $G_{2}$. The free product $G_{1} * G_{2}$ is said to be nontrivial if neither $G_{1}$ nor $G_{2}$ is the trivial group.

LEMMA 6.2. Let $\phi$ be a homomorphism of the group $G$ onto the nontrivial free product $G_{1} * G_{2}$, then $\phi$ is essential. 
In [9], J. Stallings states the following conjectures:

CONJECTURE B. Let $S$ be a closed, orientable surface of genus $n>1$. Let $F_{1}$ and $F_{2}$ be free groups of rank $n$. Let $\eta: \pi_{1}\left(S, s_{0}\right) \rightarrow F_{1} \times F_{2}$ be an epimorphism. Then there is a nontrivial element in kernel $\eta$ which may be represented by a simple closed curve in $S$ based at $s_{0}$.

CONJECTURE D. Let $G$ be the group with presentation

$$
\left\{x_{1}, y_{1}, \ldots, x_{n}, y_{n}: \prod_{i=1}^{n}\left[x_{i}, y_{i}\right]\right\}
$$

where $n>1$ and $\left[x_{i}, y_{i}\right]=x_{i}^{-1} y_{i}^{-1} x_{i} y_{i}$. Suppose $F_{1}$ and $F_{2}$ are free groups of rank $n$ and $\eta$ is a homomorphism of $G$ onto $F_{1} \times F_{2}$. Then $\eta$ can be factored through an essential map of $G$ into some free product $G_{1} * G_{2}$.

The following result due to F. Waldhausen [13] will be needed later in this section.

THEOREM (WALDHAUSEN). Let $(U, V)$ and $\left(U^{\prime}, V^{\prime}\right)$ denote Heegaard splittings of the 3-sphere $S^{3}$ having the same genus. Then there is a homeomorphism $h$ of $S^{3}$ onto itself so that $h(U)=U^{\prime}$ and $h(V)=V^{\prime}$.

REMARK. An argument is given in [9] to show that Conjecture B implies the theorem of Waldhausen stated above.

Theorem 6.3. The Poincaré Conjecture is equivalent to each of Conjecture B and Conjecture D.

Proof. An argument is given in [9, pp. 85-87], to show that Conjecture B implies Poincaré's Conjecture.

It is now shown that Poincaré's Conjecture implies Conjecture D. Let $S$ be a closed, orientable surface of genus $n>1$. Identify $\pi_{1}\left(S, s_{0}\right)$ with the group $G$ of Conjecture $\mathrm{D}$ and let $\rho_{i}$ denote the natural projection of $F_{1} \times F_{2}$ onto the $i$ th factor, $i=1,2$ [4]. If $\eta_{1}=\rho_{1} \eta$ and $\eta_{2}=\rho_{2} \eta$, then

$$
\left(\eta_{1}, \eta_{2}\right): \pi_{1}\left(S, s_{0}\right) \rightarrow F_{1} \times F_{2}
$$

is a splitting homomorphism of $\pi_{1}\left(S, s_{0}\right)$ and $\left(\eta_{1}, \eta_{2}\right)$ is identical to the homomorphism $\eta$. Let $M$ denote the 3-manifold with Heegaard splitting $(U, V)$ guaranteed in Theorem 5.2, and corresponding to the splitting homomorphism $\left(\eta_{1}, \eta_{2}\right)$ of $\pi_{1}\left(S, s_{0}\right)$ into $F_{1} \times F_{2}$. Since $\left(\eta_{1}, \eta_{2}\right)$ is an epimorphism, Lemma 6.1 says that $M$ is simply connected. Thus by Poincaré's Conjecture, $M$ is the 3-sphere $S^{3}$. The Heegaard splitting $(U, V)$ of $S^{3}$ has genus $n>1$. By Waldhausen [13], there is a simple closed curve $l$ lying in $S$ containing $s_{0}$ and separating $S$; the class, [l], determined by $l$ in $\pi_{1}\left(S, s_{0}\right)$ is not trivial; and if $\left(u_{*}, v_{*}\right)$ is the splitting homomorphism induced by $(U, V)$ then

$$
[l] \in \operatorname{kernel}\left(u_{*}, v_{*}\right)=\left(\operatorname{kernel} u_{*}\right) \cap\left(\operatorname{kernel} v_{*}\right) .
$$


Let $S^{\prime}$ denote the wedge of two surfaces obtained from $S$ by identifying $l$ to a point, and let $p$ denote the natural projection of $S$ onto $S^{\prime}$. Then $\pi_{1}\left(S^{\prime}, p\left(s_{0}\right)\right)$ $\approx G_{1} * G_{2}$, where $G_{i}(i=1,2)$ is the fundamental group of a closed, orientable surface different from the 2-sphere. Furthermore; $p_{*}$ is an epimorphism and hence by Lemma 6.2 is essential.

Since

$$
\left(\operatorname{kernel} p_{*}\right) \subset\left(\operatorname{kernel}\left(u_{*}, v_{*}\right)\right) \text {, }
$$

there is a homomorphism $\psi$ of $\pi_{1}\left(S^{\prime}, p\left(s_{0}\right)\right)$ into $\pi_{1}\left(U, s_{0}\right) \times \pi_{1}\left(U, s_{0}\right)$ so that the diagram

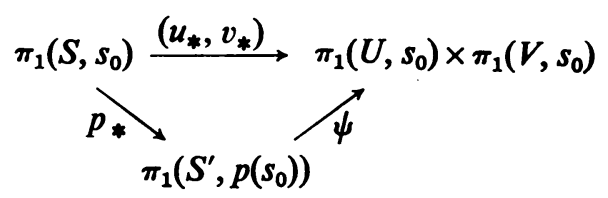

commutes. But $\left(u_{*}, v_{*}\right)$ is equivalent to $\left(\eta_{1}, \eta_{2}\right)$. Let $\alpha_{1}$ be an isomorphism of $\pi_{1}\left(U, s_{0}\right)$ onto $F_{1}$ and let $\alpha_{2}$ be an isomorphism of $\pi_{1}\left(V, s_{0}\right)$ onto $F_{2}$ so that the diagram

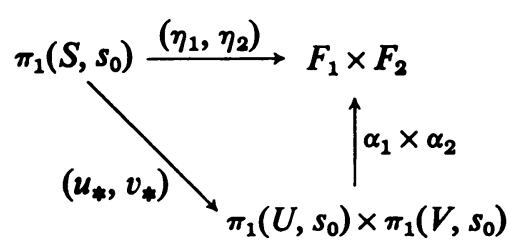

commutes. Then $\eta=\left(\eta_{1}, \eta_{2}\right)$ can be factored through $\pi_{1}\left(S^{\prime}\right)$ by the essential map $p_{*}$. In fact, $\eta=\left(\alpha_{1} \times \alpha_{2}\right) \psi p_{*}$.

The proof of Theorem 6.3 now follows from Theorem 2 of [9] which proves that Conjecture D implies Conjecture B.

\section{BIBLIOGRAPHY}

1. P. J. Hilton, An introduction to homotopy theory, Cambridge Univ. Press, London, 1964.

2. P. J. Hilton and S. Wylie, Homotopy theory, Cambridge Univ. Press, London, 1962.

3. William Jaco, Constructing three-manifolds from group homomorphisms, Doctoral Dissertation, Univ. of Wisconsin, Madison, 1968.

4. A. G. Kurosh, The theory of groups, 2nd ed., Vols. I and II, Chelsea, New York, 1960.

5. W. Mangler, Die Klassen von topologischen Abbildungen einer geschlossenen Fläche auf sich, Math. Z. 44 (1938), 541, Satz 1, 2, 542.

6. J. Nielsen, Untersuchungen zur Topologie der geschlossenen zweiseitigen Flächen, Acta Math. 50 (1927), Satz 11, 266.

7. D. R. McMillan, Jr., Homeomorphisms on a solid torus, Proc. Amer. Math. Soc. 14 (1963), 386-390. 
8. H. Seifert and W. Threlfall, Lehrbuch der Topologie, Teubner, Leipzig, 1934.

9. J. Stallings, "How not to prove the Poincare conjecture" in Topology seminar, Wisconsin, Ann. of Math. Studies, No. 60, Princeton Univ. Press, Princeton, N. J., 1965.

10. —, A topological proof of Grushko's Theorem on free products, Math. Z. 90 (1965), 1-8.

11. R. Traub, Poincaré's conjecture is implied by a conjecture on free groups, J. Res. Nat. Bur. Standard Sect. B, 71B (1967), 53-56.

12. F. Waldhausen, On irreducible 3-manifolds which are sufficiently large, Ann. of Math. 87 (1968), 56-88.

13. - Heegaard-Zerlegungen der 3-Sphäre, Topology 7 (1968), 195-203.

14. E. C. Zeeman, Seminar on combinatorial topology, Inst. Hautes Etudes Sci. Publ. Math., Paris, 1963.

15. ——, Relative simplicial approximation, Proc. Cambridge Philos. Soc. 60 (1964), 39-43.

THE UNIVERSTTY OF MichigAN, ANn Arbor, Michigan 